\title{
Modeling the potential for Zika Virus in Cairns, Australia
}

\author{
Thomas M. Kollars. Jr* \\ Department of Health Sciences, Liberty University, USA
}

\begin{abstract}
Australia is at risk of invasion from Zika virus (ZIKAV) from travelers as well as sea and air ports that serve as points of entry for infected mosquitoes and humans entering the country. The Cairns maritime port, located along the coast in northeast Australia, receives diverse international cargo and cruise ships and is at risk for the introduction of ZIKAV. The Bioagent Transport and Environmental Modeling System (BioTEMS) was used to identify an Introduction/invasion zone, a Gap infiltration zone and recommended collection sites for use within integrated mosquito management (IMM) plans. The BioTEMS was also used to model the spread of ZIKAV and validate using data from released Aedes aegypti. Based upon external validation, the BioTEMS model was accurate in predicting the distribution and directional movement of Ae. aegypti in Cairns. Recommended areas for IMM and surveillance sites are provided to assist in the mitigation or prevention of the establishment of ZIKAV through the port or by an infected person infecting local Aedes species.
\end{abstract}

Australia is at high risk to invasion by Zika virus (ZIKAV) due to its geographical proximity to the epidemic region of ZIKAV in the western Pacific [1]. There is some confusion as to how an arbovirus invades a new geographic area, whether through invasion of an infected mosquito or through importation by an infected human. Some researchers and government officials believe the route of ZIKAV introduction will be through humans infecting local mosquitoes. However, the introduction of infected mosquitoes through air or marine ports is also likely and should not be underestimated. There is sometimes failure by public health officials to recognize these two avenues. For example, in a recent CBS 60 Minutes broadcast, Dr. Anthony Fauci (the head of infectious diseases at the U.S. National Institutes of Health) stated, "The mosquito didn't fly from Rio de Janeiro to Florida. The mosquito flies 500 feet in a lifetime. It's the people who travel" [2]. However, there is absolutely no reason to rule out either or both import of ZIKAV through infected humans and/or mosquitoes, as they are both viable.

One must gather all the relevant evidence and consider all possibilities when attempting to interdict an invasive species. For example, dengue, another Flavivirus, may have been introduced in several cities in Western Australia by mosquitoes through either air or sea ports $[3,4]$. The Cairns seaport, located on the east coast of Queensland, serves cargo and cruise ships arriving from several countries in the region [5]. It is therefore at risk for invasion and subsequent spread of ZIKAV. Public health officials in Cairns would benefit by having information they could use to reduce the risk of invasion and spread of ZIKAV in Cairns as part of their integrated mosquito management (IMM) plan. The Bioagent Transport and Environmental Modeling System (BioTEMS) was used to assess the risk of invasion by ZIKAV through the port area in Cairns and provide information for IMM planning. The BioTEMS has previously been used for modeling biological weapons defense and infectious diseases in several countries and utilized for consequence management planning and during special events, e.g. presidential inauguration [6]. BioTEMS utilizes up to several hundred abiotic and biotic factors to produce risk and vulnerability assessments for biological agents and infectious diseases. Examples of biotic and abiotic factors include pathogen strain, vector/host relationship, vectorial capacity, host/ vector physiology, colonization ability, population dynamics of hosts and vectors, soil, shade, and weather conditions, such as wind, temperature, precipitation, and shade. Analytical methods within BioTEMS include artificial intelligence, fuzzy logic, niche analysis, and general additive regression. The BioTEMS model predicted ZIKAV invasion through ports in Brazil and Florida, USA, where external data was used to validate the model $[7,8]$. BioTEMS was used in the present study to provide information that public health officials can use to prevent or mitigate the effects in the event ZIKAV enters through the Cairns maritime port.

The BioTEMS, ArcMap (ESRI, Inc.), and Statistica (Quest Software, Inc.) were used to analyze geographic information and conduct data analysis. The BioTEMS TIGER model was developed to assist in identifying areas at highest risk for invasive mosquito species, associated pathogens and optimize surveillance and control efforts [9]. The acronym TIGER represents the steps in the invasion of a mosquito species or haplotype: Transport- identifies the point of origin, method and rate of transport to a locality. Introduction- identifies point or area of initial invasion/entry of species or haplotypes and preliminary spread into a locality. Gap- determines the area where vector/pathogen infiltrates and initially spreads once it has gained a foothold. Escaladeincorporates abiotic and biotic factors as possible resistance to invasion. Residence and recruitment - incorporates factors and area where vector/pathogen adds to genetic diversity or becomes endemic and recruits con-specifics/haplotypes. In predictive modeling, it is imperative to use external data to validate the model where possible $[10,11]$. In this study, external data from $[12,13]$ were used to validate the BioTEMS model for Cairns. Collection data of Aedes aegypti (L.) from the Cairns suburb was also supplied by Commonwealth of Science and Industrial Research Organisation (CSIRO). The BioTEMS and ArcView were used to produce output into Google ${ }^{\circledR}$ Earth.

Correspondence to: Thomas M. Kollars, Jr., Health Sciences, Liberty University, 1971 University Blvd, Lynchburg, VA 24515, Previous Address: Brigade Surgeon, 415th Chemical Brigade, USAR, Greenville, SC 29605, E-mail: tkollars@liberty.edu

Key words: Aedes, arbovirus, mosquito-borne disease, epidemiology, modeling

Received: May 19, 2017; Accepted: June 12, 2017; Published: June 15, 2017 
The city of Cairns appears to be at high risk for invasion by ZIKAV through the port area. Based upon validation using the external data of [13], the BioTEMS TIGER model was accurate in predicting the directional movement and distribution of Ae. aegypti in Cairns. The BioTEMS TIGER model identified an area at high risk of Introduction/ invasion of approximately $6 \mathrm{~km}^{2}$ (Figure 1). Inspection and mosquito control of incoming ships, surveillance and testing of mosquitoes, and epidemiological surveillance in the human population should be conducted in I-zone in Cairns. An area of $35 \mathrm{~km}^{2}$ was identified as the Gap zone should ZIKAV invade through the port area and infected mosquitoes disperse or personnel infect local mosquitoes. Epidemiological and mosquito surveillance/testing should be conducted in the G-zone and control measures implemented in the event ZIKAV is detected. The BioTEMS output was similar to information previously published by other researchers, supporting their conclusions as well as the output from the BioTEMS TIGER model. The BioTEMS I and G zones overlapped $95 \%$ with high density areas and breeding sites of $A e$. aegypti identified by [13] in Cairns. Recommended surveillance sites for Aedes mosquitoes were identified area wide in Cairns (Figure 1). The BioTEMS predicted a northwesterly direction of dispersal of $309^{\circ}$ should ZIKAV infected Aedes mosquitoes enter through the port in Cairns.
The distance individual Ae aegypti disperse varies geographically, from a few meters up to 3,000 meters [14,15]. In Cairns, the reported distance of dispersal of Ae aegypti, averaged $78 \mathrm{~m}$ in [12] and $94 \mathrm{~m}$ (CSIRO data). The movement of released mosquitoes was dependent on environmental factors such as trees, shade, and road barriers [12]. Using the release point in [12] as a theoretical ZIKAV affected individual or infected mosquito, the I-zone in Edge Hill encompasses 100\% of the area of the dispersed mosquitoes released in the CSIRO study. The area of the I-zone is $3.5 \mathrm{~km}^{2}$ and indicates where IMM would be conducted should such an event occur. The G-zone encompasses approximately $10 \mathrm{~km}^{2}$; mosquito surveillance sites are also identified (Figure 2). The BioTEMS model of dispersing infected Aedes mosquitoes was $99.4 \%$ similar to the mark-release-recapture study of [12] (Figure 2). The BioTEMS predicted a dispersal direction of $318^{\circ}$ for ZIKAV infected mosquitoes, whereas, the average direction of dispersal described in [12] was $316^{\circ}$. In the Edge Hill suburb, $89.2 \%$ of the mosquito capture sites in the CSIRO study were within $10 \mathrm{~m}$ of optimal surveillance sites identified using BioTEMS (Figure 2).

Another factor affecting invasion may be pesticide resistance within species and among haplotypes. For example in Malaysia, both Ae. aegypti and Ae. albopictus Skuse were shown to have variation in resistance to

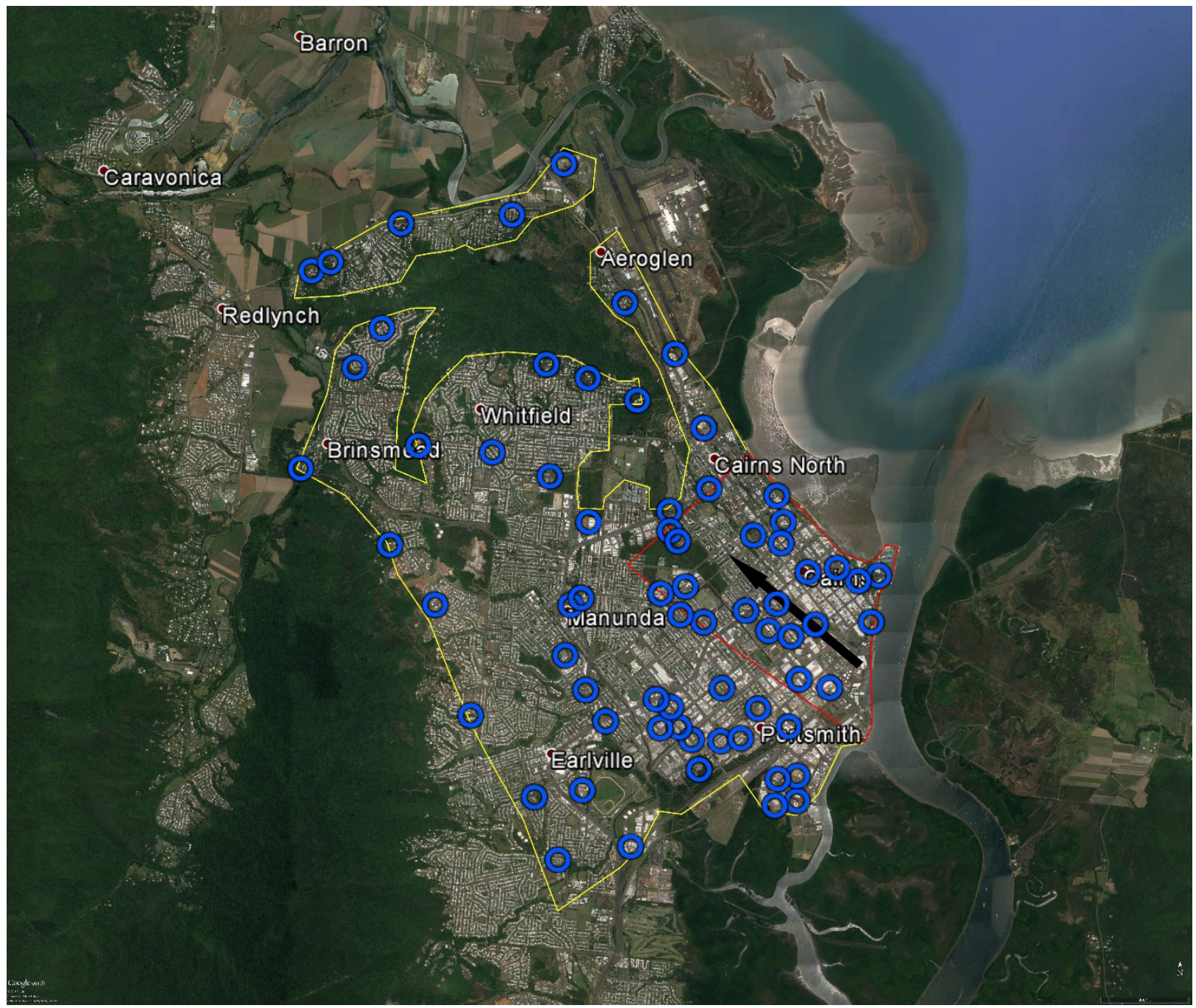

Figure 1. Map showing areas in Cairns at high risk for invasion of Zika virus (I-zone red outline) and recommended integrated mosquito management (G-zone yellow) should Zika virus be introduced through the port. Black arrow (at $309^{\circ}$ ) represents likely direction of movement if Zika virus is brought in through the port. Blue circles indicate recommended surveillance sites. 


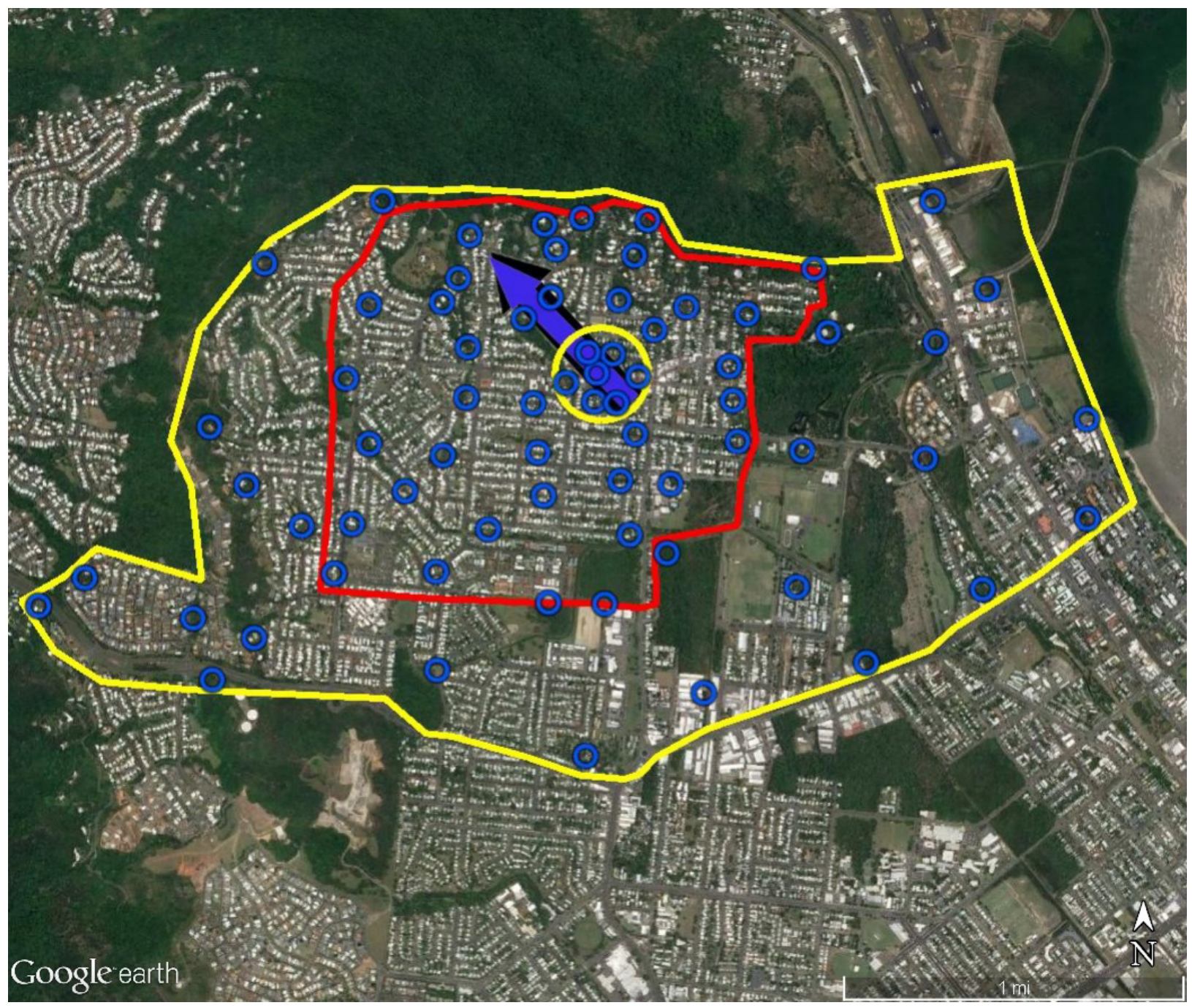

Figure 2. Map showing predicted direction of dispersal of ZIKAV infected Aedes aegypti (black arrow at $318^{\circ}$ ) and published direction of dispersal of Aedes aegypti (blue arrow at $316^{\circ}$, from Russel et al. 2005) in the Edge Hill suburb of Cairns. Blue circles indicate recommended surveillance sites. Are in yellow circle identifies areas mosquitoes were collected (data provided by Trewin Brenda). Area in red polygon identifies I-zone where immediate surveillance/control efforts should be implemented should an infected person or mosquito be identified. Area in yellow polygon identifies G-zone for surveillance to detect and mitigate spread of infected mosquitoes.

pyrethroids and malathion depending on geographic location. Ships arriving from ports where pesticide use is common, mosquito control programs must be able to test and adapt should resistant mosquitoes be detected. In addition to the importation of infected mosquito species, local Aedes may become infected through feeding on humans or having contact with infected haplotypes. If venereal and vertical transmission of ZIKAV occurs as is the case in several other arboviruses [17-20], the potential for ZIKAV to be maintained within the mosquito population makes invasion and the risk of the virus becoming endemic more likely. Public health and mosquito control officials should consider several factors when designing the IMM plan for the prevention and control of ZIKAV infected mosquitoes, e.g. species, dispersal range, pesticide resistance, seasonality, temperature, humidity, density of breeding sites, density of hosts, vector-pathogen interactions, etc. Area and direction of dispersal of Aedes within a locality should be considered in the IMM plan when possible.

In conclusion, ships arriving from cities where ZIKAV is endemic, pose a significant risk for the introduction of ZIKAV into Cairns. Should a cargo ship arriving from Malaysia contain infected mosquitoes and/or crew, the short trip of 11 days [5] may not be long enough time to clear ZIKAV infection from humans before arriving in Cairns. The crew may infect other personnel through sexual contact or they may infect local resident mosquitoes as they travel in the city. Infected Aedes mosquitoes may also invade through the port and infect human residents and possibly other conspecifics. Determining the area of an effective control zone will vary depending on locality and other abiotic and biotic factors. Public health officials should incorporate risk assessment modeling in the development of their IMM plans. Proactive preventive deployment of environmentally safe and economic pesticides may be utilized in surrounding an infected patient's home to prevent infecting local competent vector species and in port areas to reduce the risk of the establishment of invasive mosquito species/ haplotypes [9]. Expanded epidemiologic surveillance of mosquitoes and clinical/serological testing of the community living in I and G zones should be conducted. Models such as BioTEMS can be useful in assisting in IMM planning and mitigation.

\section{Acknowledgements}

The author thanks Trewin Brendan, Scott Ritchie, Dan Pagendam, and Richard Russel for providing the location of the initial release 
point and collection data from CSIRO which aided validation of the BioTEMS TIGER model. I also express gratitude to Mustapha Debboun and Gwen Sunderland for critical review of the manuscript. The views expressed in this publication are those of the author and do not reflect the official policy of the United States Army or United States Government.

\section{References}

1. Hall-Mendelin S, Pyke AT, Moore PR, Mackay IM, McMahon JL, et al. (2016) Assessment of Local Mosquito Species Incriminates Aedes aegypti as the Potential Vector of Zika Virus in Australia. PLoS Negl Trop Dis 10: e0004959. [Crossref]

2. LaPook J (2016) What's being done to fight the Zika virus? http://www.cbsnews.com/ news/60-minutes-zika-in-the-united-states-mosquito-disease.

3. Whelan P, Nguyen H, Hajkowicz K, Davis J, Smith D, et al. (2012) Evidence in Australia for a case of airport dengue. PLoS Negl Trop Dis 6: e1619. [Crossref]

4. Lindsay MD, Jardine A, Giele C, Armstrong P, McCarthy S, et al. (2015) Investigation of the First Case of Dengue Virus Infection Acquired in Western Australia in Seven Decades: Evidence of Importation of Infected Mosquitoes? PLoS Negl Trop Dis 9: e0004114. [Crossref]

5. Ports North (2016) Cruise ships arrivals and departures. www.portsnorth.com.au/ourports/cruise-arr-dep.php

6. Kollars TM (2008) BioTEMS-Biology based modeling to determine bioagent fate. Chemical Biological Weapons Delivery Methods and Consequence Assessment Modeling Conference. National Geospatial Intelligence Center: Weapons Intelligence, Nonproliferation and Arms Control.

7. Kollars TM Jr., JW Kollars (2016) The Invasion of Zika Virus into Rio De Janeiro and Fortaleza, Brazil, Inside Out or Outside In? Ann Com Med Prac 2: 1015-1018.

8. Kollars TM (2017) Assessing likely invasion sites of Zika virus infected mosquitoes and naval maritime ports in Florida. Res Rep Trop Med 8: 1-6.

9. Kollars TM Jr., Kollars PG, B Hulsey (2016) Reducing the Risk to Marine Ports from Invasive Mosquito Species, Zika, Dengue, Chikungunya viruses and Filariasis. Int $J$ Med 4: 70-73.
10. Justice AC, Covinsky KE, Berlin JA (1999) Assessing the generalizability of prognostic information. Ann Intern Med 130: 515-524. [Crossref]

11. Ahmed I, Debray TP, Moons KG, Riley RD1 (2014) Developing and validating risk prediction models in an individual participant data meta-analysis. BMC Med Res Methodol 14: 3. [Crossref]

12. Russell RC, Webb CE, Williams CR, Ritchie SA (2005) Mark-release-recapture study to measure dispersal of the mosquito Aedes aegypti in Cairns, Queensland, Australia. Med Vet Entomol 19: 451-457. [Crossref]

13. Duncombe J, Clements A, Davis J, Hu W, Weinstein P, et al. (2013) Spatiotempora patterns of Aedes aegypti populations in Cairns, Australia: assessing drivers of dengue transmission. Trop Med Int Health 18: 839-849. [Crossref]

14. Reiter P, Amador MA, Anderson RA, Clark GG (1995) Short report: dispersal of Aedes aegypti in an urban area after blood feeding as demonstrated by rubidium-marked eggs. Am J Trop Med Hyg 52: 177-179. [Crossref]

15. Honório NA, Silva Wda C, Leite PJ, Gonçalves JM, Lounibos LP, et al. (2003) Dispersal of Aedes aegypti and Aedes albopictus (Diptera: Culicidae) in an urban endemic dengue area in the State of Rio de Janeiro, Brazil. Mem Inst Oswaldo Cruz 98: 191-198. [Crossref]

16. Ishak IH, Jaal Z, Ranson H, Wondji CS (2015) Contrasting patterns of insecticide resistance and knockdown resistance (kdr) in the dengue vectors Aedes aegypti and Aedes albopictus from Malaysia. Parasit Vectors 8: 181. [Crossref]

17. Rosen L (1987) Sexual transmission of dengue viruses by Aedes albopictus. Am J Trop Med Hyg 37: 398-402. [Crossref]

18. Diallo M, Thonnon J, Fontenille D (2000) Vertical transmission of the yellow fever virus by Aedes aegypti (Diptera, Culicidae): dynamics of infection in F1 adult progeny of orally infected females. Am J Trop Med Hyg 62: 151-156. [Crossref]

19. Mavale M, Parashar D, Sudeep A, Gokhale M, Ghodke Y, et al. (2010) Venereal transmission of chikungunya virus by Aedes aegypti mosquitoes (Diptera: Culicidae) Am J Trop Med Hyg 83: 1242-1244. [Crossref]

20. Thangamani S, Huang J, Hart CE, Guzman H, Tesh RB (2016) Vertical Transmission of Zika Virus in Aedes aegypti Mosquitoes. Am J Trop Med Hyg 95: 1169-1173. [Crossref]

Copyright: (C2017 Kollars TM. This is an open-access article distributed under the terms of the Creative Commons Attribution License, which permits unrestricted use, distribution, and reproduction in any medium, provided the original author and source are credited. 